\title{
Fermi Glass Versus High-Temperature Superconductivity Behavior
}

\author{
Müller, K A
}

\begin{abstract}
Very recently BaSrNiO4 was reported to be a Fermi glass (Schilling et al. in J. Phys., Condens. Matter 21:015701, 2009). Its structure is essentially the one of $\mathrm{K} 2 \mathrm{NiF} 4$ as is that of $\mathrm{La} 2 \mathrm{CuO} 4$, in which the occurrence of high-temperature superconductivity (HTS) upon hole doping was first reported (Bednorz and Müller in Z. Phys.B 64:189, 1986; Adv. Chem. 100:757, 1988). The carriers in both have mainly eg character, and move in a stochastic potential as documented by a number of experiments. The difference of the two behaviors is mainly ascribed to the formation of intersite bipolarons (Kabanov and Mihailovic in J. Supercond. 13:950, 2000), which is estimated to be up to two orders of magnitude larger in La2CuO4 than in BaSrNiO4. From this it follows that for HTS to occur, a large bipolaron formation energy in layered structures is required
\end{abstract}

DOI: https://doi.org/10.1007/s10948-010-0769-x

Posted at the Zurich Open Repository and Archive, University of Zurich ZORA URL: https://doi.org/10.5167/uzh-156690

Journal Article

Published Version

Originally published at:

Müller, K A (2010). Fermi Glass Versus High-Temperature Superconductivity Behavior. Journal of Superconductivity and Novel Magnetism, 23(7):1225-1228.

DOI: https://doi.org/10.1007/s10948-010-0769-x 


\title{
Fermi Glass Versus High-Temperature Superconductivity Behavior
}

\author{
K.A. Müller
}

Received: 16 February 2010 / Accepted: 17 February 2010 / Published online: 22 April 2010

(C) Springer Science+Business Media, LLC 2010

\begin{abstract}
Very recently $\mathrm{BaSrNiO}_{4}$ was reported to be a Fermi glass (Schilling et al. in J. Phys., Condens. Matter 21:015701, 2009). Its structure is essentially the one of $\mathrm{K}_{2} \mathrm{NiF}_{4}$ as is that of $\mathrm{La}_{2} \mathrm{CuO}_{4}$, in which the occurrence of high-temperature superconductivity (HTS) upon hole doping was first reported (Bednorz and Müller in Z. Phys. B 64:189, 1986; Adv. Chem. 100:757, 1988). The carriers in both have mainly $\mathrm{e}_{\mathrm{g}}$ character, and move in a stochastic potential as documented by a number of experiments. The difference of the two behaviors is mainly ascribed to the formation of intersite bipolarons (Kabanov and Mihailovic in J. Supercond. 13:950, 2000), which is estimated to be up to two orders of magnitude larger in $\mathrm{La}_{2} \mathrm{CuO}_{4}$ than in $\mathrm{BaSrNiO}_{4}$. From this it follows that for HTS to occur, a large bipolaron formation energy in layered structures is required.
\end{abstract}

Keywords Fermi glass vs. high-temperature superconductivity

The discovery of superconductivity in $\mathrm{La}_{2-x} \mathrm{Me}_{x} \mathrm{CuO}_{4}$, with $\mathrm{Me}=\mathrm{Ba}$, Sr etc., [4, 5] led to a worldwide effort in materials science to find new doped ionic compounds with enhanced superconducting transition temperatures $T_{\mathrm{c}}$. In one avenue of research, compounds were synthesized in which the $\mathrm{CuO}_{2}$ planes were maintained. Others looked for entirely new layered crystals. In the former the so far highest $T_{\mathrm{c}}$ of $133.5 \mathrm{~K}$ at ambient pressure was found [22]. In the latter effort, the picnides were discovered, in which a maximum $T_{\mathrm{c}}$ of $56 \mathrm{~K}$ was reported [8, 21], still below the boiling point

K.A. Müller $(\varangle)$

Physik-Institut der Universität Zürich, Winterthurerstr. 190, 8057

Zurich, Switzerland

e-mail: irmamf@bluewin.ch of liquid nitrogen. In between these possibilities is the one in which the perovskite-type $\mathrm{MeO}_{2}$ plane is maintained, but the $\mathrm{Cu}^{2+}$ ion is replaced by another transition-metal ion. The $\mathrm{Ni}^{3+}$ ion was a promising candidate because its low-spin configuration $3 \mathrm{~d}^{7}$ is $\mathrm{t}_{2 \mathrm{~g}}^{6}$, $\mathrm{e}_{\mathrm{g}}^{1}$. This means that $\mathrm{Ni}^{3+}$ has one Jahn-Teller $\mathrm{e}_{\mathrm{g}}$ electron, like the $\mathrm{Cu}^{2+}$ ion has one $\mathrm{e}_{\mathrm{g}}$ hole. It was known that $\mathrm{LaSrNiO}_{4}$, in which $\mathrm{Ni}$ is trivalent, is a metal. However, Demazeau et al. [10] found that $\mathrm{LaBaNiO}_{4}$ with its enlarged lattice exhibits variable range hopping (VRH). Therefore, Schilling et al. [23] more recently investigated the mixed crystal series $\mathrm{LaSr}_{1-x} \mathrm{Ba}_{x} \mathrm{NiO}_{4}$ for the occurrence of HTS. The series starting with $x=1$ and VRH became more conducting with decreasing $x$, with $x=0$ being a metal, i.e., an Anderson transition. For $\mathrm{BaLaNiO}_{4}$ $(x=1)$, susceptibility, specific heat, and thermal conductivity indicated a near constant electron density at the Fermi energy, which means that it is a Fermi glass as originally predicted by Anderson [2, 3].

To understand the entirely different behaviors of, say, $\mathrm{La}_{2-x} \mathrm{Sr}_{x} \mathrm{CuO}_{4}$, i.e., when partially replacing the $\mathrm{La}^{3+}$ with two-valent ions, such as $\mathrm{Sr}^{2+}$, and $\mathrm{BaLaNiO}_{4}$, we have to go back to the first experimental observation of a Fermi glass. This was reported for $\mathrm{CaV}_{2} \mathrm{O}_{5}$ doped with protons [19]. The electrons introduced to keep charge neutrality reduce the valency of $\mathrm{V}$ from $5+$ to $4+$, yielding a $3 \mathrm{~d}^{1+}$ state. For high enough dopings, the electrons become itinerant and exhibit VRH, i.e., a conductivity with a $T^{-1 / 4}$ dependence as reproduced in Fig. 1a.

Because of the mobile carriers one electron spin resonance (ESR) line with Lorentzian line shape is detected, which upon integration yields the paramagnetic susceptibility $\chi(T)_{\mathrm{p}}$ of the carriers (multiplied by $T$ because of the Curie law). This is reproduced in Fig. 1b. From it one sees that $\chi(T)_{\mathrm{p}}$ is independent of the temperature from 


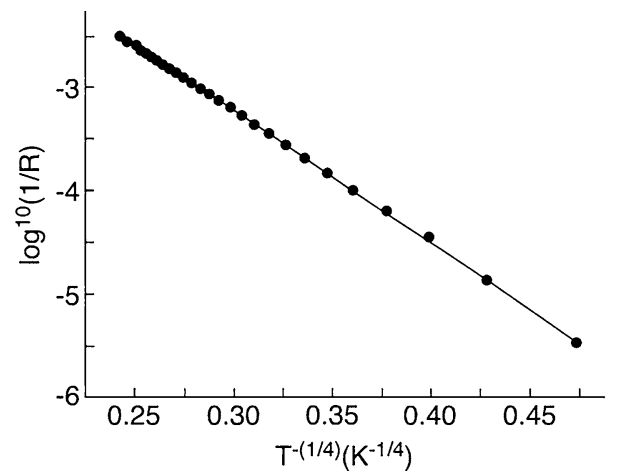

Fig. 1a The inverse resistivity plotted logarithmically as a function of $T^{-1.4}$ for a $\mathrm{H}: \mathrm{CaV}_{2} \mathrm{O}_{6}$ sample heated for $20 \mathrm{~h}$ at $470{ }^{\circ} \mathrm{C}$. $\sigma_{0}=3.95 \Omega^{-1} \mathrm{~cm}^{-1}$ and $T_{0}^{1 / 4}=29.7 \mathrm{~K}^{1 / 4}$ [see Ref. [19]]

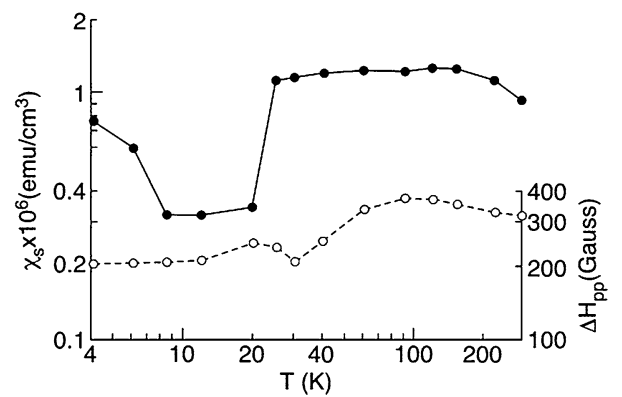

Fig. 1b Conduction-electron spin resonance line susceptibility and width of $\mathrm{H}: \mathrm{CaV}_{2} \mathrm{O}_{5}$ as a function of $\log T$ for the same sample as in Fig. 1a. Because of macroscopic inhomogeneities, the scale of the spin susceptibility is only approximate (see Ref. [19])

$300 \mathrm{~K}$ down to $20 \mathrm{~K}$ in the range where VRH is observed. This is what had been predicted for a Fermi glass by Anderson [2, 3]. Below $20 \mathrm{~K}, \chi(T)_{\mathrm{p}}$ drops to a considerably lower value. This occurred for all samples tested at the time, and it was commented upon in the last paragraph of [19]: The reproducible drop in $\chi_{\mathrm{p}}$ below $20 \mathrm{~K}$ could also result from negative electron correlation-energy of the order of $10 \mathrm{~K}$, which tends to pair the electrons.

We can now compare the Fermi-glass response found in $\mathrm{CaV}_{2} \mathrm{O}_{5}$ to that in $\mathrm{BaLaNiO}_{4}$. In Fig. 2a, the temperature dependence of the resistivity and in Fig. $2 b$ that of the specific heat are reproduced from [23]. Over the low temperature range where VRH is observed, $\chi(T)_{\mathrm{p}}$ was not constant upon cooling but increased slightly and was analyzed by an expression $\chi(T)_{\mathrm{p}}=C /(T+\Theta)+\chi_{0}$, with either $\Theta=0$ or $\Theta \neq 0$. In the former, $C=2.4 \times 10^{-2} \mathrm{emu} \mathrm{K} \mathrm{mol}^{-1}$ and $\chi_{0}=4.5 \times 10^{-4} \mathrm{emu} \mathrm{mol}^{-1}$ and in the latter case $C=5.4 \times$ $10^{-2} \mathrm{emu} \mathrm{K} \mathrm{mol}^{-1}$ and $\chi_{0}=3.4 \times 10^{-4} \mathrm{emu} \mathrm{mol}^{-1}$, i.e., a substantial temperature-independent paramagnetic term. No drop in $\chi(T)_{\mathrm{p}}$ can be seen, but at low temperatures the data scatter quite a bit. Therefore, in Fig. $2 b$ the specific-heat data are reproduced below $16 \mathrm{~K}$. In these, no clear deviation from the fit of a $C(T) / T=\beta T^{2}+\gamma$ is detectable. Here the elec-

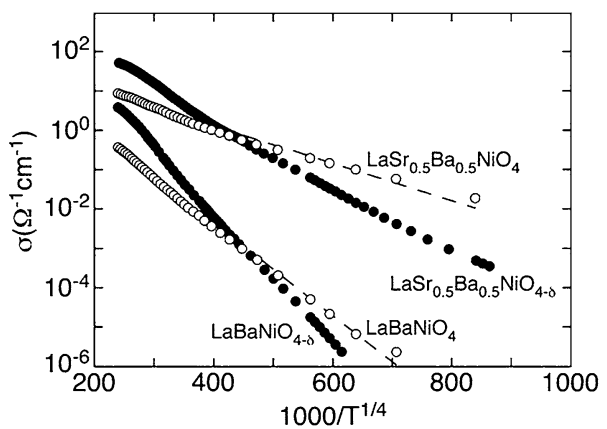

Fig. 2a Electrical conductivities $\sigma$ versus $T^{-1 / 4}$ of as-prepared samples (full circles) and of high-pressure oxygen-annealed samples (open circles). The dashed lines are fits according to a variable-range hopping-type conductivity (see Ref. [23])

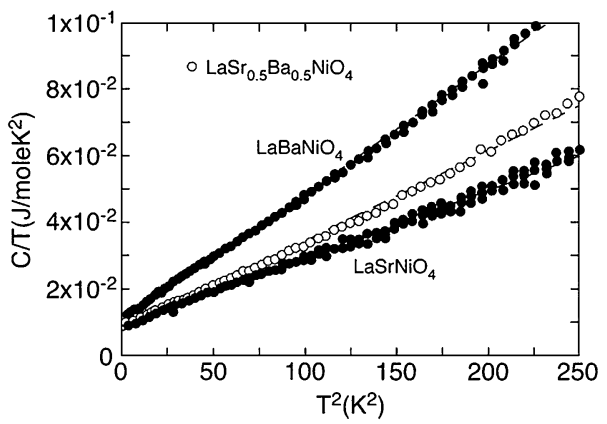

Fig. 2b Reduced specific heat $C / T$ versus $T^{2}$ data of $\mathrm{LaSrNiO}_{4}$ (bottom), oxygen treated $\mathrm{LaSr}_{0.5} \mathrm{Ba}_{0.5} \mathrm{NiO}_{4}$ (middle) and $\mathrm{LaBaNiO}_{4}$ (upper curve). The dashed lines are fits to $C(T)=\beta T^{3}+\gamma T$ (see Ref. [23])

tronic term $\gamma$ equals $9.5 \mathrm{~mJ} / \mathrm{mol} \mathrm{K}$. Therefore, there no sign of bipolaron formation appears to be present.

This behavior has to be verified by integrating over the motionally narrowed ESR line of the $\mathrm{Ni}^{3+}$ as a function of temperature to obtain $\chi(T)_{\mathrm{p}}$. The $\mathrm{Ni}^{3+}$ ESR line has been observed earlier [10]. If also in $\chi(T)_{\mathrm{p}}$ no drop occurs, then the bipolaron formation energy is lower than that in $\mathrm{H}: \mathrm{CaV}_{2} \mathrm{O}_{5}$, and in both one to two orders of magnitude lower than in the low doping range of $\mathrm{La}_{2-x} \mathrm{Sr}_{x} \mathrm{CuO}_{4}$. In the latter compound, the electron paramagnetic resonance (EPR) of probing $\mathrm{Mn}^{2+}$ ions yielded a bipolaron formation energy of 460(40) $\mathrm{K}$ independent of doping for $x$ from 1 to $6 \%$, see Fig. 3 [24, 25]. A comparison of Fig. 1b, Fig. 2b and Fig. 3 indicates that for a small bipolaron formation energy a Fermi glass and for a large one HTS results. In all three cases the carrier concentration is clearly inhomogeneous, that is, metallic clusters or stripes and more isolating areas are present, as it follows from the VRH or EPR [10, 23-25].

From this experimental interpretation, the question naturally arises why the energy for bipolaron formation is about two orders of magnitude larger in $\mathrm{La}_{2} \mathrm{CuO}_{4}$ than in $\mathrm{BaLaNiO}_{4}$. In $\mathrm{La}_{2-x} \mathrm{Sr}_{x} \mathrm{CuO}_{4}$ there are two holes with an- 

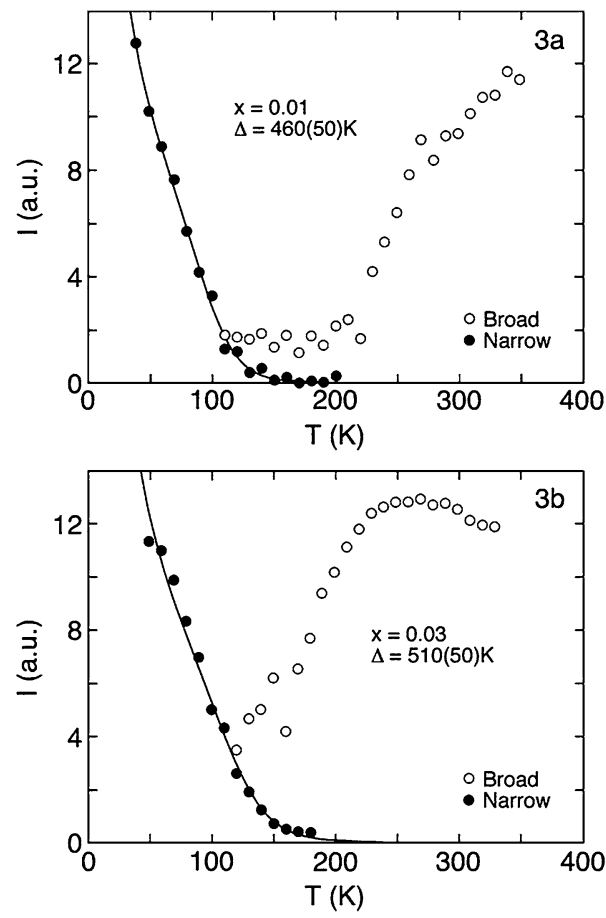

Fig. 3 Temperature dependence of the narrow and broad EPR signal intensity in $\mathrm{La}_{2-x} \mathrm{Sr}_{x} \mathrm{Cu}_{0.98} \mathrm{Mn}_{0.02} \mathrm{O}_{4}$ with different $\mathrm{Sr}$ dopings: (a) $x=0.01$; (b) $x=0.03$. The solid lines represent fits using the model described in Ref. [24, 25]

tiparallel spin having their density mainly located on oxygens near two $\mathrm{Cu}^{2+}$ (with an oxygen connecting them). The trapping occurs because the oxygens surrounding these $\mathrm{Cu}^{2+}$ ion sites are locally shifted by a Jahn-Teller $\mathrm{e}_{\mathrm{g}}$ conformation [15, 17]. Essential is the density of the two holes on the oxygens where calculations from first principles yielded a formation energy between 100 and $700 \mathrm{~K}$ [16] comparable with that of $460 \mathrm{~K}$ found by EPR $[24,25]$. On the other hand, in $\mathrm{BaLaNiO}_{4}$ the carriers in question are located mainly on $3 \mathrm{~d}, \mathrm{e}_{2 \mathrm{~g}}$ orbitals, at least at low temperatures [10]. Apart from hybridization, a spreading of these electrons onto oxygens would render the charge of the latter more negative than two. However, this is not known to occur, quite in contrast to the opposite, namely, the lowering of the oxygen charge as it occurs in hole-doped $\mathrm{La}_{2} \mathrm{CuO}_{4}$. We believe that because of this more local charge on the $\mathrm{Ni}^{3+}$ in $\mathrm{BaLaNiO}_{4}$ the bipolaron formation is hindered. Finally in proton-doped $\mathrm{CaV}_{2} \mathrm{O}_{6}$, the energy for bipolaron formation lies in between the ones of the compounds just discussed. Here the donated electrons are mainly located at the vanadium ions, forming $\mathrm{V}^{4+}$, as observed by EPR. Owing to the higher valency, the hybridization is larger than for $\mathrm{Ni}^{3+}$, and a larger bipolaron energy formation appears than in $\mathrm{BaLaNiO}_{3}$.

The above discussion has left out the dependence of the bipolaron binding energy on the degree of doping as was first theoretically investigated by Alexandrov, Kabanov and Mott (AKM) [1]. In this important paper, AKM calculated

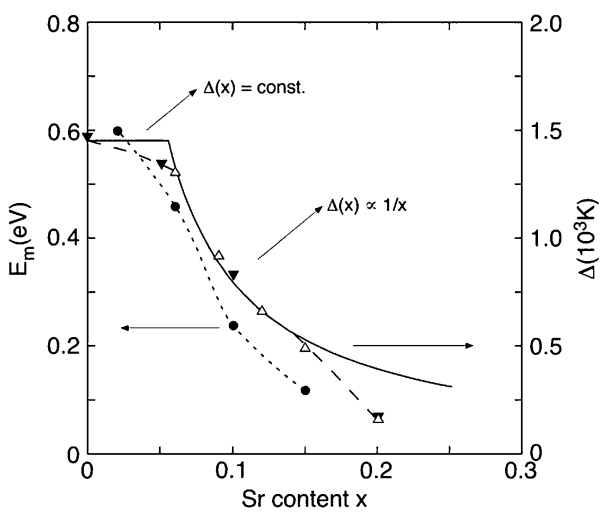

Fig. 4 Composition dependences of the bipolaron binding energy $\Delta$ and $E_{\mathrm{m}}=2 \mathrm{~g}^{2} \mathrm{~h} \omega$. The left-hand and right-hand scales are for the circle and triangle symbols, respectively. $\Delta$ is proportional to $1 / x$ for $0.06<x<0.15$. The solid triangles represent $T_{\max }$ as determined by Johnston [14]. The results for $E_{\mathrm{m}}$ are from reference [6]

the bipolaron binding energy as a function of the carrier concentration $x$, see Fig. 4. For low $x$, the binding energy is constant up to $x_{\mathrm{c}}$ (as observed by EPR [24, 25]), and from there it decreases as $1 / x$. This was confirmed by the analysis of existing susceptibility data by Müller et al. [20]. In order to get the analysis self-consistent they added a temperatureindependent Pauli term, see Fig. 4. The latter is present because of the metallic clusters found by EPR already for low dopings $[24,25]$. Note also that in photoemission this was later seen as density of states at the $\mathrm{X}$ and $\mathrm{M}$ points of the Brillouin zone, i.e., Fermi arcs and bipolaron states, respectively [9]. Towards the optimum doping near $x=0.15$, the bipolaronic binding energy is lowered by a factor of $\approx 6$, see Fig. 4. However it appears that it does not vanish. This can be concluded from the doping dependence of the pseudogap temperature $T^{*}$, where the bipolaron formation occurs upon cooling, see Fig. 13 in [18]. In contrast to theories where at optimum doping a quantum critical point exists, requiring $T^{*}(x)$ to vanish, $T^{*}(x)$ remains finite as verified by tunneling [11], inelastic neutron scattering [13], and EPR [18] measurements. Therefore, the bipolaron binding energy stays finite as well. The above addressed bipolaron binding near optimum doping at $x=0.15$ is $\Delta \simeq 70 \mathrm{~K}$ (Fig. 4), whereas in $\mathrm{LaBaNiO}_{4}$ the concentration is $x=1$. Here the bipolaron binding may well be nil, despite the fact that it is finite for $x \approx 0.15$. For this doping the two-band polaronic model of Bussmann-Holder and Keller [7] is more appropriate.

In [23], the reason for the occurrence of a stochastic potential in $\mathrm{LaBaNiO}_{4}$ or, in other words, the rising of the mobility edge to the Fermi level such that VRH occurs, was not addressed. From the incomplete oxygenation of their samples, Demazeau et al. [10] argued that $\mathrm{Ni}^{2+}$ ions may be present. However in [23] highly oxygenated samples exhibited a very clear VRH, see Fig. 1a. What was not considered in [23] and [10] is the presence of high- and low-spin $\mathrm{Ni}^{3+}$ 
as the origin of stochasticity due to local lattice deformation because of the different size of the high- and low-spin $\mathrm{Ni}^{3+}$ [12]. This is well documented in Fig. 3 of [10]. The high-spin signal reduces substantially upon cooling, and at $4.2 \mathrm{~K}$ is barely visible. However, it may still be responsible for the Curie law term $C=2.4$ to $5.4 \times 10^{-2} \mathrm{emu} \mathrm{K} \mathrm{mol}^{-1}$ in the susceptibility [23], and thus warrants a detailed EPR investigation. We also note that in the normal samples of $\mathrm{LaBaNiO}_{4}$, VRH is less well followed (a straight line in the $T^{-1 / 4}$ plots) than in $\mathrm{H}_{2} \mathrm{Ca}_{2} \mathrm{~V}_{5} \mathrm{O}_{4}$, see Fig. $1 \mathrm{a}$.

Acknowledgements The author is indebted to A. Schilling, A. Bussmann-Holder, and H. Keller for various discussions and a critical reading of the manuscript, further to T. Crudeli for his help to produce the figures.

\section{References}

1. Alexandrov, A.S., Kabanov, V.V., Mott, N.F.: Phys. Rev. Lett. 77, 4796 (1996)

2. Anderson, P.W.: Phys. Rev. 109, 1492 (1958)

3. Anderson, P.W.: Comments Solid State Phys. 2, 193 (1970)

4. Bednorz, J.G., Müller, K.A.: Z. Phys. B 64, 189 (1986)

5. Bednorz, J.G., Müller, K.A.: Adv. Chem. 100, 757 (1988)

6. Bi, X.X., Eklund, P.C.: Phys. Rev. Lett. 70, 2625 (1993)

7. Bussmann-Holder, A., Keller, H.: Eur. Phys. J. B 44, 487 (2005)

8. Chen, X.H., Wu, T., Wu, G., Liu, R.H., Chen, H., Fang, D.F.: Nature 453, 761 (2008)
9. Damascelli, A. Hussein, Z., Shen, Z.X.: Rev. Mod. Phys. 75, 473 (2003)

10. Demazeau, G., Marty, J.L., Buffet, B., Dance, J.M., Pouchard, M., Dordor, P., Chevalier, B.: Mat. Res. Bull. 17, 37 (1982)

11. Deutscher, G.: Rev. Mod. Phys. 77, 109 (2005)

12. Filippi, M., Kundys, B., Agrestini, S., Prellier, W., Oyanagi, H., Saini, N.L.: J. Appl. Phys. 106, 104116 (2009)

13. Furrer, A.: In: Superconductivity in Complex Systems. Structure and Bonding, vol. 114, p. 171. Springer, Berlin (2005). Fig. $16 \mathrm{c} \& \mathrm{~d}$

14. Johnston, D.C.: Phys. Rev. Lett. 62, 957 (1989)

15. Kabanov, V.V., Mihailovic, D.: J. Supercond. 13, 950 (2000)

16. Kochelaev, B.I., Safina, A.M., Shengelaya, A.S., Müller, K.A., Conder, K.: Mod. Phys. Lett. B 17, 415 (2003)

17. Mihailovic, D., Kabanov, V.V.: Phys. Rev. B 63, 054505 (2001)

18. Müller, K.A.: J. Phys., Condens. Matter 19, 251002 (2007)

19. Müller, K.A., Penny, T., Shafer, M.W., Fitzpatrick, W.J.: Phys. Rev. Lett. 47, 138 (1981)

20. Müller, K.A., Zhao, G.M., Conder, K., Keller, H.: J. Phys., Condens. Matter 10, L291 (1998)

21. Ren, Z.A., Yang, J., Lu, W., Yi, W., Shen, X.L., Li, Z.C., Che, G.C., Dong, X.L., Sun, L.L., Zhou, F., Zhao, Z.X.: Europhys. Lett. 82, 57002 (2008)

22. Schilling, A., Cantoni, M., Ott, H.R.: Nature 337, 56 (1993)

23. Schilling, A., Dell'Amore, R., Karpinski, J., Bukowski, Z., Medarde, M., Pomjakushina, E., Müller, K.A.: J. Phys., Condens. Matter 21, 015701 (2009)

24. Shengelaya, A.S.: Tbilisi State University, Thesis (2005)

25. Shengelaya, A.S., Brun, M., Kochelaev, B.I., Safina, A.M., Conder, K., Müller, K.A.: Phys. Rev. Lett. 93, 017001 (2004) 